\title{
Peptide Receptor Radionuclide Therapy as a Novel Treatment for Metastatic and Invasive Phaeochromocytoma and Paraganglioma
}

\author{
Ingrid Y.F. Mak Aimee R. Hayes Bernard Khoo Ashley Grossman \\ Neuroendocrine Tumour Unit, ENETS Centre of Excellence, Royal Free Hospital, London, UK
}

\section{Keywords}

Peptide receptor radionuclide therapy - MIBG .

Phaeochromocytoma - Paraganglioma - Therapy .

Metastatic

\begin{abstract}
At present there is no clinical guideline or standardised protocol for the treatment of metastatic or invasive phaeochromocytoma and paraganglioma (collectively known as PPGL) due to the rarity of the disease and the lack of prospective studies or extended national databases. Prognosis is mainly determined by genetic predisposition, tumour burden, rate of disease progression, and location of metastases. For patients with progressive or symptomatic disease that is not amenable to surgery, there are various palliative treatment options available. These include localised therapies including radiotherapy, radiofrequency, or cryoablation, as well as liver-directed therapies for those patients with hepatic metastases (e.g., transarterial chemoembolisation) and systemic therapies including chemotherapy or molecular targeted therapies. There is currently intense research interest in the value of radionuclide therapy for neuroendocrine tumours, including phaeochromocytoma and paraganglioma, with either iodine-131 ( ${ }^{131}$ I)-radiolabelled metaiodobenzylguanidine or very recently peptide receptor radionuclide therapy
\end{abstract}

\section{KARGER}

() 2019 S. Karger AG, Basel

E-Mail karger@karger.com

www.karger.com/nen
(PRRT), and the most important contemporary clinical studies will be highlighted in this review. The studies to date suggest that PRRT may induce major clinical, biochemical, and radiological changes, with ${ }^{177}$ Lu-DOTATATE being most efficacious and presenting less toxicity than ${ }^{90} \mathrm{Y}$-DOTATATE. Newer combination therapies with combined radioisotopes, or combinations with chemotherapeutic agents, also look promising. Given the favourable efficacy, logistic, and safety profiles, we believe that PRRT will probably become the standard treatment for inoperable metastatic PPGL in the near future, but we await data from definitive randomised controlled trials to understand its role.

(c) 2019 S. Karger AG, Basel

\section{Introduction}

Metastatic phaeochromocytoma (PCC) and paraganglioma (PGL; collectively known as PPGL) is a challenging disease entity as existing therapeutic options are limited. The 5-year overall survival (OS) rate ranges from 34 to $69 \%[1,2]$. Around $34 \%$ of all patients with PPGL will harbour a germline mutation, with succinate dehydrogenase (SDH) subunit B (SDHB; 10.3\%), SDHD (8.9\%), VHL (7.3\%), RET (6.3\%), and NF1 (3.3\%) being most prevalent [3]. PGL have a higher malignant potential, 
with up to $35 \%$ of patients being reported to have metastases [4-6] ( $>40 \%$ if SDHB mutation positive [3]). The location of the primary tumour in PGL is often not amenable to complete surgical removal, thus leading to a worse prognosis. A tumour size $>5 \mathrm{~cm}$, elevated plasma and/or urine methoxytyramine levels, a pathological Phaeochromocytoma of the Adrenal gland Scaled Score (PASS) $>6$ for PCCs only, loss of tumoural SDHB staining on immunohistochemistry, and a Ki-67 proliferation index $>3 \%$, are other predictive factors for metastatic disease [1]. Traditionally, "malignancy" is operationally defined as the development of metastatic lesions from a PCC or PGL in non-chromaffin tissues, as there are no definitive histopathological criteria for predicting biological aggressiveness in PPGL. However, this terminology is no longer advocated according to the latest 2017 World Health Organisation classification [7]. The most common sites of metastasis are regional lymph nodes, bones, liver and lungs [1]: patients with visceral metastases tend to have a worse prognosis than those with isolated bone disease [8].

The principal management goals in metastatic PPGL are to control symptoms related to catecholamine excess, improve quality of life, prolong survival, and reduce other complications (such as pathological fracture). Debulking or cytoreductive surgery may decrease catecholamine secretion and potentially enhance the efficacy of other therapies [9]; however, the overall value of cytoreductive surgery remains uncertain in the setting of metastatic PPGL due to lack of comparative trials with systemic or locoregional therapies. Interestingly, in a recent retrospective series of 53 patients with PPGL and synchronous metastases, those patients who had resection of the primary tumour had a longer median OS compared to those who did not ( 85 vs. 36 months, $p<0.001$ ), irrespective of other factors such as primary tumour size and location as well as number of metastases [10].

For inoperable cases with progressive or symptomatic disease, there are a variety of localised or systemic palliative treatments available. External beam radiotherapy can be useful for the local control of bone lesions, with symptomatic relief in $94 \%$ of the lesions with tumour-related symptoms, and a local control rate of $81 \%$ at 5 years in a recent retrospective cohort involving 107 treatment sites in 41 patients [11].

With regards to systemic therapies, conventional chemotherapy with cyclophosphamide, vincristine and dacarbazine (CVD) seems to be effective and relatively welltolerated in the short term, but early relapses are expected [1]. A meta-analysis of four small retrospective studies in- cluding 50 metastatic PPGL patients treated with CVD revealed complete responses, partial responses and stable disease in 4,37 , and $14 \%$ respectively [12]. No improvement in OS was demonstrated in one long-term follow-up study [13], although small series have suggested better response rates in the more aggressive SDHB mutation-related PPGL cohort $[2,14]$. Similarly, partial responses to temozolomide have been specifically observed in SDHB-related tumours [15]. In terms of molecular targeted therapy, sunitinib was retrospectively shown to produce clinical benefit concerning tumour size, uptake on ${ }^{18} \mathrm{~F}$-FDG-PET scanning, disease stabilisation and blood pressure control in 17 patients (8 with SDHB mutations) [16], and is currently being explored in the ongoing randomised phase II study, FIRST-MAPPP (NCT01371201). The effectiveness of cabozantinib was evaluated in a small phase II trial; the objective response rate was $45 \%$ with a progression-free survival (PFS) of 11 months, while $91 \%$ of patients reported clinical benefit [17]. Other potential systemic therapies for SDHx-related PPGL, including the DNA methyl transferase inhibitor guadecitabine (NCT03165721) and PARP inhibitors, are currently under investigation.

\section{Results on Radionuclide Therapy}

\section{Conventional ${ }^{131} I-M I B G$}

Historically, the major radionuclide used in the therapy of PPGLs has been iodine-131 $\left({ }^{131} \mathrm{I}\right)$-radiolabelled metaiodobenzylguanidine $\left({ }^{131} \mathrm{I}-\mathrm{MIBG}\right.$, also known as iobenguane), principally in use from the 1980s. ${ }^{131}$ I-MIBG, a high-energy beta-particle-emitting iodinated guanidine analogue structurally comparable to noradrenaline (norepinephrine), selectively concentrates in tissues expressing the cell membrane noradrenaline reuptake and vesicular monoamine transporters with high avidity, e.g., tumours of neuroectodermal origin and the sympathoadrenal system $[18,19]$. A recent meta-analysis of observational studies, combining the data of 243 patients with metastatic PPGLs treated with ${ }^{131}$ I-MIBG therapy, showed that 3,27 , and $52 \%$ had a complete response, partial response and stable disease, respectively, although there was marked heterogeneity in the response assessment between the studies [20]. In addition, hormonal responses were reported as complete, partial, and stable in 11,40 , and $21 \%$, respectively. These findings are in accord with the conclusion from earlier studies that at least partial tumour responses could be expected in $24-45 \%$ of patients after ${ }^{131}$ I-MIBG therapy $[9,21]$, with potential anti-hypertensive effects [22]. 
Fig. 1. A 54-year-old male with SDHB mutation-positive metastatic PCC presenting with right adrenal mass $(72 \times 59 \mathrm{~mm})$ and skeletal disease who had an ${ }^{123}$ I-MIBG with SPECT (anterior planar image; a), ${ }^{68} \mathrm{Ga}$ DOTATATE-PET/CT (maximal intensity projection [MIP] image; b), and ${ }^{18} \mathrm{~F}-\mathrm{FDG}$ PET/CT (MIP image; c) performed within 2 months of each other. ${ }^{123}$ I-MIBG shows a non-avid right adrenal mass with no other sites of disease identified (a); ${ }^{68} \mathrm{Ga}$-DOTATATE-PET/CT (b) and ${ }^{18}$ F-FDG-PET/ CT (c) identify the large right adrenal mass in addition to multifocal bony metastases, best characterised on ${ }^{68} \mathrm{Ga}$-DOTATATEPET/CT. The majority of skeletal disease and the right adrenal mass have intense ${ }^{68} \mathrm{Ga}$-DOTATATE avidity (greater than background liver), thus making the patient eligible for PRRT. Courtesy of Dr. Shaunak Navalkissoor, Department of Nuclear Medicine, Royal Free Hospital, London, UK, and Dr. Saira Reynolds, Department of Nuclear Medicine, Broomfield Hospital, Chelmsford, UK.

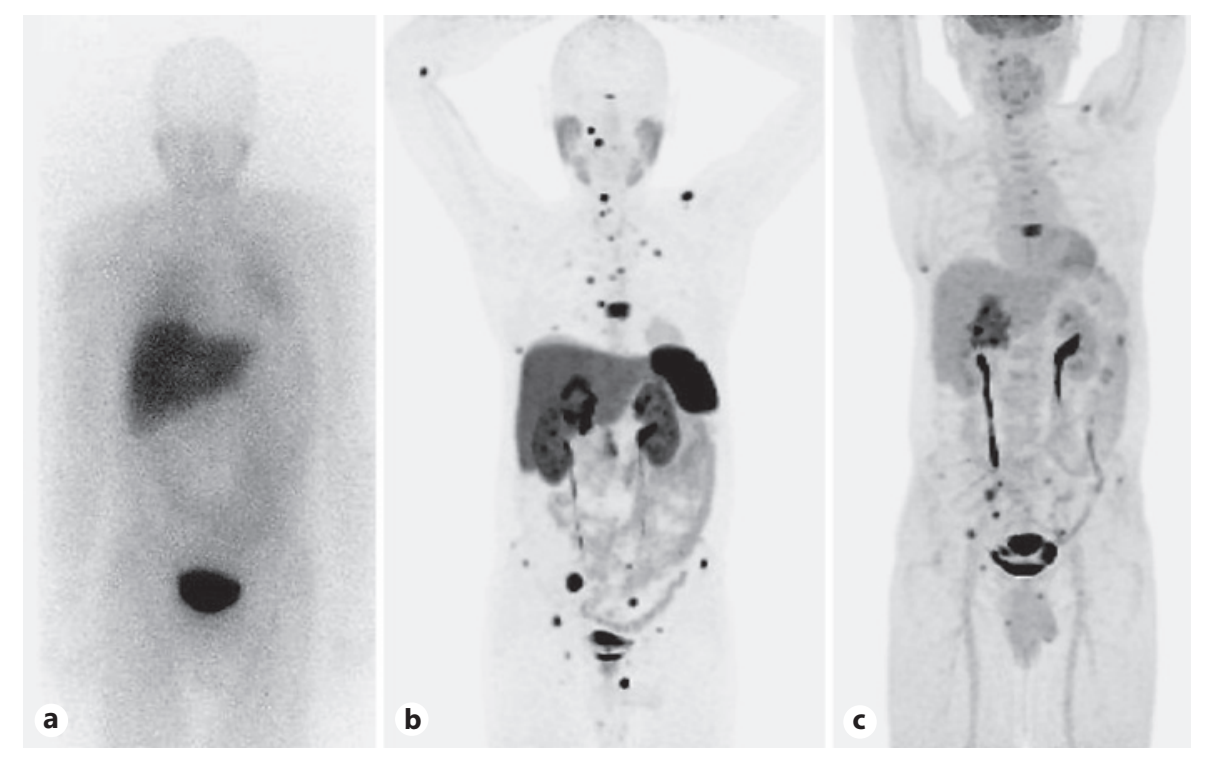

Some authors have suggested that limited sessions of high-dose and multiple low-dose ${ }^{131} \mathrm{I}$-MIBG treatments are both effective in inducing symptomatic control and disease stabilisation in patients with metastatic PPGL [19]. Although a high-dose regimen (single doses of 18$43 \mathrm{GBq}$ ) demonstrated a disease control rate of $69 \%$ in a previous phase II study [23], the risk of severe myelosuppression was high, with grade $3 / 4$ neutropaenia and thrombocytopaenia reported in 87 and $83 \%$ respectively; 4 patients required autologous haematopoietic stem cell rescue and, concerningly, 2 developed acute myeloid leukaemia during long-term follow-up. Furthermore, the reported 5-year OS rate (64\%) was similar to conventionaldose ${ }^{131}$ I-MIBG studies [24].

Hypothyroidism is another well-known side effect, and therefore the use of potassium iodide for protection of the thyroid gland is required in the peri-procedural period. Other adverse events include hypertension, gastrointestinal upset, liver toxicity, renal impairment, and gonadal failure, warranting close monitoring and regular long-term follow-up $[9,24,25]$. Certain medications that interfere with MIBG uptake such as labetalol, calcium channel blockers, tricyclic antidepressants, sympathomimetic amines (phenylephrine, ephedrine), and serotoninnoradrenaline reuptake inhibitors may need to be withdrawn prior to treatment to optimise uptake. The long duration of isolation after radiation exposure is also a drawback of conventional ${ }^{131}$ I-MIBG therapy $[9,19]$.

\section{High-Specific Activity ${ }^{131}$ I-MIBG}

Very recently, the FDA has approved Azedra ${ }^{\circledR}$ (Progenics Pharmaceuticals), a high-specific-activity preparation of ${ }^{131} \mathrm{I}-\mathrm{MIBG}$, as the first and only approved therapy for patients over 12 years old with MIBG-avid, unresectable, locally advanced or metastatic PPGL. As the transporter mechanisms on which MIBG action relies are a saturable pathway, theoretically high-specific-activity labelling should allow for greater uptake and retention of the radiopharmaceutical. The FDA approval was based on the data from a pivotal phase II open-label, multicentre trial in which 68 patients with MIBG-positive metastatic PPGL, who were not candidates for curative surgery, received one or two therapeutic doses of Azedra ${ }^{\circledR}$ : $25 \%$ of the patients reached the primary endpoint of $\geq 50 \%$ reduction of all blood pressure-lowering medications for at least 6 months, while 23 and 69\% achieved a partial response and stable disease, respectively, by response evaluation criteria in solid tumours (RECIST), constituting a disease control rate of $92 \%$ [26]. The median OS was 37 months and the most frequent $(\geq 50 \%)$ treatment-emergent adverse events were bone marrow suppression, nausea, and fatigue. 


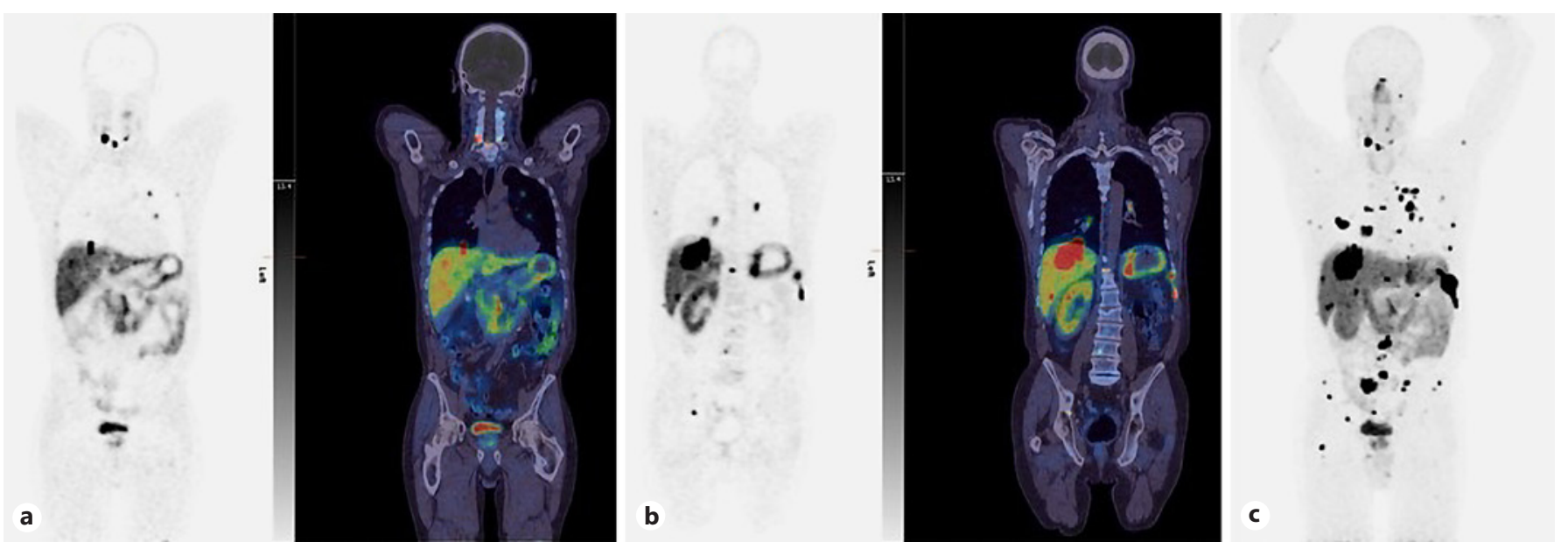

Fig. 2. Recurrent PCC following resection of primary in a 58-year-old male (PPGL susceptibility gene testing normal). Maximal intensity projection (MIP) and coronal images show extensive ${ }^{68}$ Ga-DOTATATE avid disease in liver, lung, nodes, and bone, with significant disease progression at baseline (a), 6 months (b), and 12 months (c) despite treatment. Courtesy of Dr Shaunak Navalkissoor, Department of Nuclear Medicine, Royal Free Hospital, London, UK.

\section{Peptide Receptor Radionuclide Therapy}

Sufficient lesion avidity on ${ }^{123}$ I-MIBG scintigraphy is an important prerequisite for therapeutic ${ }^{131}$ I-MIBG. Although ${ }^{123}$ I-MIBG scanning has a sensitivity of around $90 \%$ in detecting PPGL in general, this drops to $<80 \%$ for metastatic lesions $[1,27]$ and even lower $(<50 \%)$ for SDHx-associated tumours and head-and-neck PGLs (HNPGL) [1]; this is due to reduced expression of norepinephrine transporters in these mutations, indicating inadequate MIBG avidity in these conditions and ruling out MIBG therapy in many cases. Peptide receptor radionuclide therapy (PRRT), using radiolabelled somatostatin analogues, may serve as an appealing and possibly preferable alternative [28]. In fact, the majority of PPGL, like other neuroendocrine tumours (NETs), strongly express somatostatin receptors (SSTRs) of high affinity and specificity [29-31], with the most common subtypes being $2 \mathrm{a}$ and $3[32,33]$. Indeed, many metastatic PPGL demonstrate high uptake on SSTR-based scans but not on ${ }^{123}$ I-MIBG scintigraphy [34-36]. It has also been observed that SDHx-deficient tumour cells exhibit much stronger SSTR immunostaining when compared to SDHx-sufficient tumours [37, 38]. Several other studies have illustrated the diagnostic superiority of SSTRbased imaging for patients with SDHB mutations and HNPGLs [39-42], and also MEN2-associated metastatic PCC [43], due to some degree of functional dedifferentiation in these more aggressive tumours [18]. ${ }^{68} \mathrm{Ga}-\mathrm{DO}$ TA-somatostatin analogue-PET scanning was shown to be the best diagnostic imaging modality for metastatic PPGL, with significantly higher sensitivity (92-100\%) and greater lesion-to-background contrast when compared to ${ }^{123}$ I-MIBG scintigraphy, ${ }^{18} \mathrm{~F}-\mathrm{DOPA}-\mathrm{PET}$, or ${ }^{18}$ F-FDG-PET [3, 36, 42, 44, 45], and thus should be considered as the investigation of choice to identify disease extent (Fig. 1), although with the caveat that small PCC may be difficult to distinguish against normal adrenal uptake and adjacent renal uptake. Of all modern SSTRbased imaging techniques, ${ }^{68} \mathrm{Ga}$-DOTATATE-PET scanning is most favoured as it has the greatest affinity for SSTR type 2 [30] (Fig. 2). Although unlabelled "cold" somatostatin analogues have been notably successful for gastroenteropancreatic NETs, these have been unsuccessful in the control of the hormonal syndrome in metastatic PPGLs [9]. A well-designed study involving 10 patients with PCC failed to demonstrate efficacy of monthly intramuscular octreotide in symptomatic or hormonal control [46]. This may possibly be explained by the speculation that metastatic PCCs, as opposed to metastatic PGLs, are mostly SSTR type 2 negative [47]. This may also relate to the fact that octreotide lacks affinity for SSTR type 3 and newer multiligand agents such as pasireotide may theoretically be more effective [33, 48, 49], but this concept has not as yet been tested.

There is a growing body of evidence that PRRT using high-energy beta-particle-emitting radionuclides such as ${ }^{90} \mathrm{Y}$-DOTATOC and ${ }^{177} \mathrm{Lu}$-DOTATATE (Fig. 3) is highly effective in the treatment of NETs in terms of objective 
Fig. 3. A 28-year-old female with head and neck PGL (SDHB mutation-positive and variant of unknown significance in SDHD gene; primary tumour resected) metastatic to lung and bone with ${ }^{68} \mathrm{Ga}$-DOTATATE avidity. ${ }^{68} \mathrm{Ga}$-DOTATATE maximal intensity projection (MIP) image before treatment (a); she was treated with PRRT ( ${ }^{177} \mathrm{Lu}$-DOTATATE), and anterior view (b) and posterior view (c) of the whole body post-treatment scans show a similar distribution of radioisotope uptake. Courtesy of Dr Shaunak Navalkissoor, Department of Nuclear Medicine, Royal Free Hospital, London, UK.

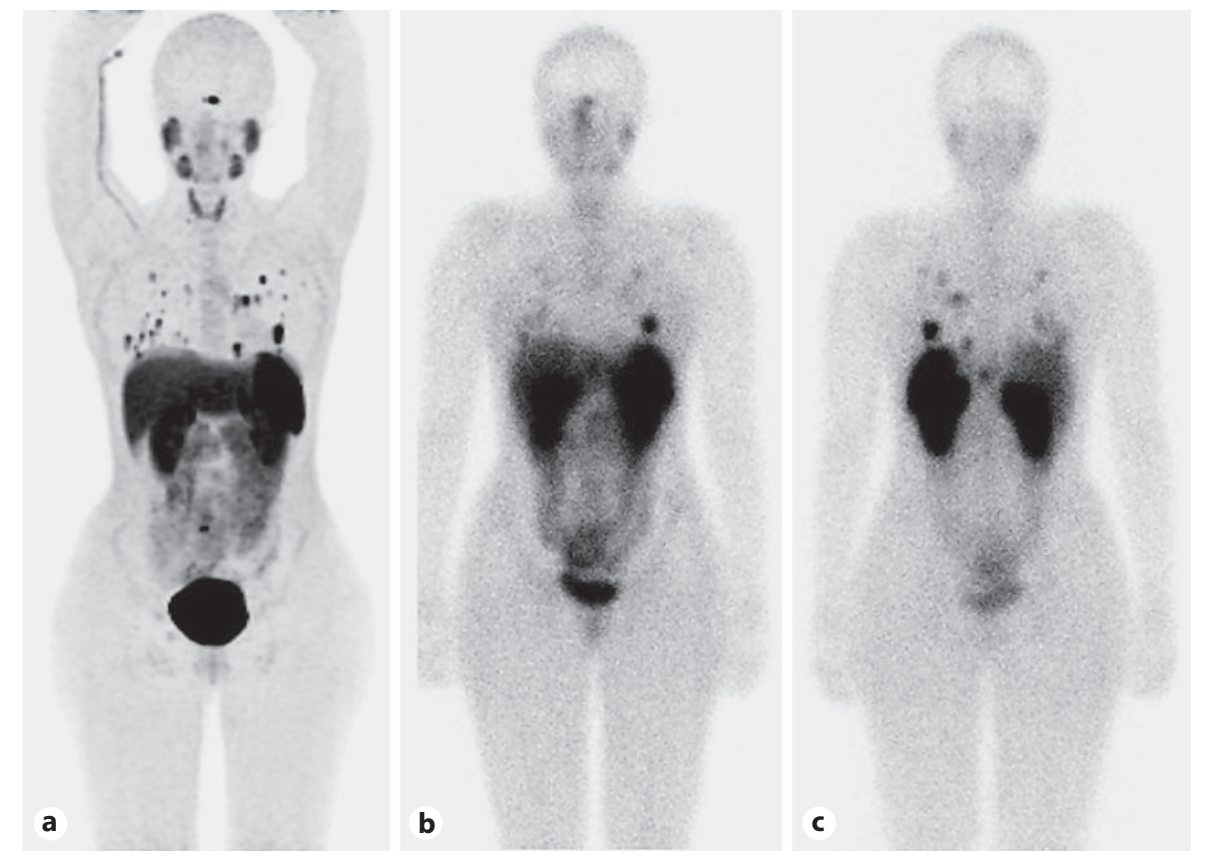

tumour response, disease stabilisation, symptomatic control, and preservation of quality of life, while maintaining a favourable safety profile [50-52]. This was specifically demonstrated in the phase III randomised controlled trial NETTER-1, in which treatment with ${ }^{177} \mathrm{Lu}$-DOTATATE resulted in a significantly longer PFS and a higher objective response rate when compared to high-dose octreotide LAR among patients who had metastatic midgut NETs [50]. There was a 79\% reduction in the risk of disease progression or death with ${ }^{177} \mathrm{Lu}$-DOTATATE $(p<$ 0.001). In light of these impressive findings, the FDA has approved Lutathera ${ }^{\circledR}\left({ }^{177} \mathrm{Lu}\right.$-DOTATATE) for the treatment of SSTR-expressing gastroenteropancreatic NETs in adults, while in the UK the National Institute for Health and Care Excellence (NICE) has assessed PRRT as costeffective for the treatment of this subgroup of NETs.

Around a decade ago, some centres first reported their experience in using PRRT for metastatic PPGL. In a cohort of 20 patients with metastatic NETs, Frilling et al. [53] illustrated a case of an unresectable metastatic HNPGL showing a partial response after ${ }^{90} \mathrm{Y}$-DOTATOC and ${ }^{177} \mathrm{Lu}$-DOTATOC treatments. In another case series, 12 patients with PGL (75\% metastatic) received ${ }^{177} \mathrm{Lu}$-DOTATATE; of the 4 patients who had evidence of disease progression at baseline, 2 demonstrated tumour regression and 1 stable disease after treatment, whereas more than half of the remaining patients had stable disease after a median follow-up of 13 months [54]. Later, in a retrospective phase II study, Forrer et al. [34] described 28 metastatic PPGL with radiological progression at baseline (9 PCCs, 19 PGLs) showing long-lasting partial remissions after ${ }^{90} \mathrm{Y}$-DOTATOC (3 combined with ${ }^{177} \mathrm{Lu}$ DOTATOC), with only limited and tolerable toxicities. Partial remissions, minor responses, and stable disease were observed in 2,5 , and 13 patients, respectively, at the time of restaging. These effects were quite durable with a mean duration of $18 \pm 14$ months, with the two partial remissions lasting 23 and 41 months, respectively. However, it is worth noting that the tumour characteristics, imaging, and treatment protocols involved in these studies were heterogeneous due to their retrospective nature, and the subjects had undergone various treatment modalities before PRRT. Subsequently, the only prospective phase II clinical trial was conducted in a single centre to examine the efficacy and safety profile of ${ }^{90} \mathrm{Y}$-DOTATOC, recruiting 1,109 patients with metastatic NETs, including 11 PCCs and 28 PGLs [55]. All patients had disease progression before study entry. Radiographic, biochemical, and clinical responses were observed in 36.4, 18.2 , and $45.5 \%$, respectively, in the PCC group, and 10.7, 14.3 , and $21.4 \%$, respectively, among the PGL patients. The PCC and PGL groups had a mean OS of 32 and 82 months, respectively. Another important finding in this large cohort was that tumour uptake on the initial molecular imaging correlated with OS (HR 0.45, $p<0.01$ ). Overall, there were 142 patients (12.8\%) showing tran- 
sient grade 3-4 myelosuppression, while 102 patients (9.2\%) had severe permanent renal toxicity.

More recently published papers have further explored the use of PRRT in patients with surgically incurable disease associated with SDHx mutations and HNPGLs. Zovato et al. [56] studied 4 female SDHD carriers with biochemically silent, non-metastatic carotid body or thoracic PGL, considered inoperable due to the tumour size or location, who later developed disease progression and received 3-5 cycles of ${ }^{177} \mathrm{Lu}-\mathrm{DOTATATE}$. Two showed a partial response by RECIST while the other 2 had stable disease with reduced tumour uptake on subsequent scintigraphy. Another case report described a patient carrying an SDHD mutation with a history of a resected cervical PGL presenting with histologically proven bone and liver metastases, strongly avid on ${ }^{68} \mathrm{Ga}$-DOTATOC-PET/ CT but with negative ${ }^{124}$ I-MIBG-PET/CT scanning [57]. These lesions showed minor responses after two cycles of ${ }^{90}$ Y-DOTATOC. Pinato et al. [28] examined the efficacy of ${ }^{177} \mathrm{Lu}$-DOTATATE in 5 young patients with aggressive, metastatic SDHB-related intra-abdominal PGL, all with bone metastases and catecholamine excess: 3 patients with metastatic relapse after prior excision achieved stabilisation following PRRT, 1 had a partial response, while 1 patient progressed. The median PFS was 17 months while the median OS was not reached. Another case series reported 9 patients with unresectable HNPGL, either due to critical tumour location or multifocal involvement, undergoing ${ }^{90} \mathrm{Y}$-DOTATATE PRRT (4 in combination with ${ }^{177} \mathrm{Lu}$-DOTATATE) [58]. It is unclear whether these cases had progressive or stable disease at baseline. After PRRT, all patients had stable disease by RECIST. Of the two-thirds presenting with symptoms related to cranial nerve or bone involvement, symptomatic improvement after treatment correlated with the imaging response in 4 cases. Only 1 patient had distant metastases (rib, vertebra, and lung) before PRRT, and there was marked reduction in $\mathrm{SUV}_{\max }$ from 27.6 to 5.4 in the spinal lesions after treatment, in concordance with the resolution of back pain. Another patient had resolution of tinnitus due to reduction in tumour size. Remarkable improvements were also seen in the case of a young man with a recurrent unresectable carotid body PGL, as reported by Gupta et al. [59]: he had a response at the primary site and total disappearance in the nodal and T7 vertebral metastases after five cycles of ${ }^{177} \mathrm{Lu}$-DOTATATE. Cecchin et al. [60] also described a patient aged in his thirties presenting with multifocal non-secretory PGL without a known germline mutation who underwent PRRT after presenting with worsening symptoms of spi- nal cord compression due to progressive spinal disease. His symptoms subsided with a $70 \pm 13.0 \%$ mean volume reduction in his 23 spinal lesions on post-treatment MRI scans; the mean $\mathrm{SUV}_{\max }$ reduction in 17 lesions was $85 \pm$ $13.3 \%$ (5 were no longer visible) on ${ }^{68} \mathrm{Ga}$-DOTANOC$\mathrm{PET} / \mathrm{CT}$ transaxial images. In a retrospective study concerning 14 patients with HNPGLs who underwent three cycles of ${ }^{177} \mathrm{Lu}-D O T A T A T E$, Estêvão et al. [61] found that patients with jugulotympanic PGLs achieved better treatment outcomes than those with carotid body lesions in terms of symptoms and SUV on ${ }^{68} \mathrm{Ga}$-DOTANOC$\mathrm{PET} / \mathrm{CT}$, and the initial tumour SUV actually predicted the subsequent response to PRRT $(R=+0.64, p=0.014)$. None of the patients suffered from major treatment-related adverse events in the above studies. It is also of some interest that responsivity to ${ }^{177} \mathrm{Lu}$-DOTATATE may be demonstrated using functional biochemical assessment; using proton- 1 magnetic resonance spectroscopy $\left({ }^{1} \mathrm{H}\right.$ MRS), Casey et al. [62] have recently shown that there was a loss of the succinate peak, a metabolic marker of the SDH enzymatic defect, after 4 cycles of ${ }^{177} \mathrm{Lu}-\mathrm{DO}-$ TATATE treatment in a single case, although the significance of this change on future prognosis is unclear.

A recent retrospective analysis focused on the potential effects on clinical, biochemical, and tumour responses, and survival benefits of PRRT in 20 patients (mutation status: 7 SDHB, 1 SDHD, 2 negative, 10 unknown; primary location: 8 PCC, 12 PGL) having either uncontrolled hypertension due to functional PPGL or nonfunctional progressive metastatic disease or recurrence [35]: $80 \%$ of these patients $(16 / 20)$ had not received prior systemic therapy. One to four courses of ${ }^{177} \mathrm{Lu}$-DOTATATE PRRT were administered with a median cumulative dose of $22 \mathrm{GBq}$. Nine patients received radiosensitising chemotherapy with 5-fluorouracil and/or temozolomide. At 3 months after treatment, 8/13 patients (62\%) with refractory secondary hypertension were able to reduce the dose of anti-hypertensive medications, 8/13 reported symptomatic benefit, and 4/7 who had elevated plasma metanephrine/normetanephrine measurements showed reduced levels; $86 \%(12 / 14)$ had serial declines in chromogranin A. Of those with sufficient data, 47, 41, and $12 \%$, and 36,50 , and $14 \%$, had an imaging response, stable findings or progressive disease on post-treatment ${ }^{68} \mathrm{Ga}$-DOTATATE-PET/CT $(n=17)$ and CT scans $(n=$ 14), respectively. The median PFS was 39 months while the median OS was not reached (median follow-up, 28 months). It is, however, important to note that the treatment indication for the majority of the study cohort was uncontrolled secondary hypertension rather than radio- 
logical disease progression. The most common toxicities were grade 2-3 lymphopaenia and thrombocytopaenia. Symptoms of acute catecholamine release developed $3 \mathrm{~h}$ after ${ }^{177} \mathrm{Lu}$-DOTATATE administration in 1 patient without adequate alpha-adrenoceptor blockade and with a possible contribution from dexamethasone premedication. Another patient died of unexplained cardiac arrest shortly after the third cycle of PRRT.

These results, albeit in small series and case reports with heterogeneous patient groups and treatment outcome measurements, clearly demonstrate that PRRT is a strikingly promising treatment option for metastatic as well as localised but inoperable PPGL, with substantial tumour responses and symptomatic relief, and minimal toxicity. Responses are seen in patients with multiple lesions, SDHB/D mutations and bone involvement, irrespective of the primary tumour location.

\section{Comparative Radionuclide Studies}

To date, very few studies have compared the performances of different radiopharmaceutical agents in the treatment of PPGLs. In a recent retrospective study conducted at our centre, PRRT appeared to produce a better treatment response and greater survival benefit but comparable side-effect profiles when compared to ${ }^{131}$ I-MIBG therapy [63]. In this cohort comprising 22 patients with progressive, metastatic PPGLs (7 PCC and 15 PGL, 5 SDHB-related), 9 received PRRT $\left(8{ }^{90} \mathrm{Y}\right.$-DOTATATE, 1 ${ }^{177} \mathrm{Lu}$-DOTATATE), $11{ }^{131} \mathrm{I}$-MIBG, and 2 a combination of the above. A total of 30 treatment cycles were administered; 9 treatments ( $8{ }^{131} \mathrm{I}-\mathrm{MIBG}, 1$ PRRT) to the PCC group and 21 treatments ( ${ }^{131} \mathrm{I}-\mathrm{MIBG}, 13$ PRRT) to the PGL group. Most PCCs were treated with ${ }^{131}$ I-MIBG due to reasonable uptake on MIBG scans, whereas PGLs were treated with either ${ }^{131}$ I-MIBG or PRRT based on their increased avidity on SSTR-based imaging. Considering all patients in the study cohort, PRRT demonstrated higher rates of disease stabilisation (as per RECIST assessment) when compared to ${ }^{131} \mathrm{I}-\mathrm{MIBG}(13 / 13$ vs. $10 / 16, p=0.013)$, but there was no significant difference in either mean OS ( $60.8 \pm 11.1$ vs. $41.2 \pm 10.4$ months, $p=0.09)$ or mean PFS $(38.5 \pm 9.4$ vs. $20.6 \pm 3.4$ months, $p=0.10)$. However, when the treatment outcome data regarding ${ }^{131}$ I-MIBG and PRRT were further stratified according to tumour type, there was a significant difference in OS and PFS in the PGL group in favour of PRRT ( $22.8 \pm 6.3$ vs. $60.8 \pm 11.1$ months, $p=0.012$, and $14.4 \pm 2.9$ vs. $38.5 \pm 9.4$ months, $p=0.018$, respectively). The rate of disease stabilisation in the PRRT arm was also superior to ${ }^{131} \mathrm{I}-\mathrm{MIBG}$ in the PGL cohort ( $13 / 13$ vs. $4 / 8, p=0.005)$. These results suggest that

PRRT and Phaeochromocytomas/PGLs
PGL may show improved outcomes with PRRT compared with ${ }^{131}$ I-MIBG. There has indeed been speculation that PCC and PGL may each display different biological and biochemical characteristics, with metastatic PGLs having much higher affinity for SSTR type 2 than metastatic PCCs [47]. However, given the small number of patients with PCC receiving PRRT in this study, no conclusions could be drawn. Moreover, half of the patients (11/22) had additional systemic therapies such as CVD chemotherapy and somatostatin analogues, confounding the effects of PRRT. More carefully designed and balanced, larger-scale prospective trials are therefore required before such conclusions can be definitively drawn. There were no major differences in toxicities between the two treatment arms. While there is a much longer experience with "conventional" ${ }^{131}$ I-MIBG and renal toxicity may be less frequent [19], PRRT has the potential benefits of producing fewer haematological side effects and does not require pre-treatment thyroid blockade [35]. However, the choice must also be governed by tracer concentration in tumour lesions in the respective nuclear imaging modalities [35].

DOTATATE is currently the most widely used DOTA-conjugated somatostatin analogue for PRRT as it binds with 6- to 9-fold greater affinity to SSTR type 2 compared with DOTATOC $[64,65]$. Based on the current literature, ${ }^{177} \mathrm{Lu}$-DOTA-somatostatin analogue-PRRT has at least similar efficacy in the treatment of various NETs when compared to ${ }^{90} \mathrm{Y}[66]$, but its toxicity profile is much more favourable in terms of myelosuppression and nephrotoxicity $[17,66-68] .{ }^{177} \mathrm{Lu}$ emits beta particles with a shorter range, lower energy but longer half-life when compared to ${ }^{90} \mathrm{Y}$, and it also emits gamma radiation which allows post-treatment monitoring of tumour uptake and dosimetry $[64,65]$. To date, there is no study specifically comparing the efficacy of these two radionuclides in the treatment of PPGLs. Nevertheless, given their different radiobiological properties, some researchers have evaluated the clinical outcomes of combination therapy $[69,70]$. Prasad et al. [71] retrospectively analysed three different treatment regimens among $20 \mathrm{pa}-$ tients with metastatic PPGL $\left(7^{90} \mathrm{Y}\right.$-DOTATATE, $5^{177} \mathrm{Lu}$ DOTATATE, 8 sequential combination of the two) and found that the response rate was highest $(50 \%)$ in the combination group (vs. 14 and $20 \%$ in the ${ }^{90} \mathrm{Y}$ and ${ }^{177} \mathrm{Lu}$ group, respectively): $29 \%(2 / 7)$ and $57 \%(4 / 7)$ had stable lesions and progressive disease, respectively, in the ${ }^{90} \mathrm{Y}$ group, as compared to $60 \%(3 / 5)$ and $20 \%(1 / 5)$, respectively, in the ${ }^{177}$ Lu group. However, this study is small and observational, and few data are available on their methods or toxicity. Larger prospective studies are required to 


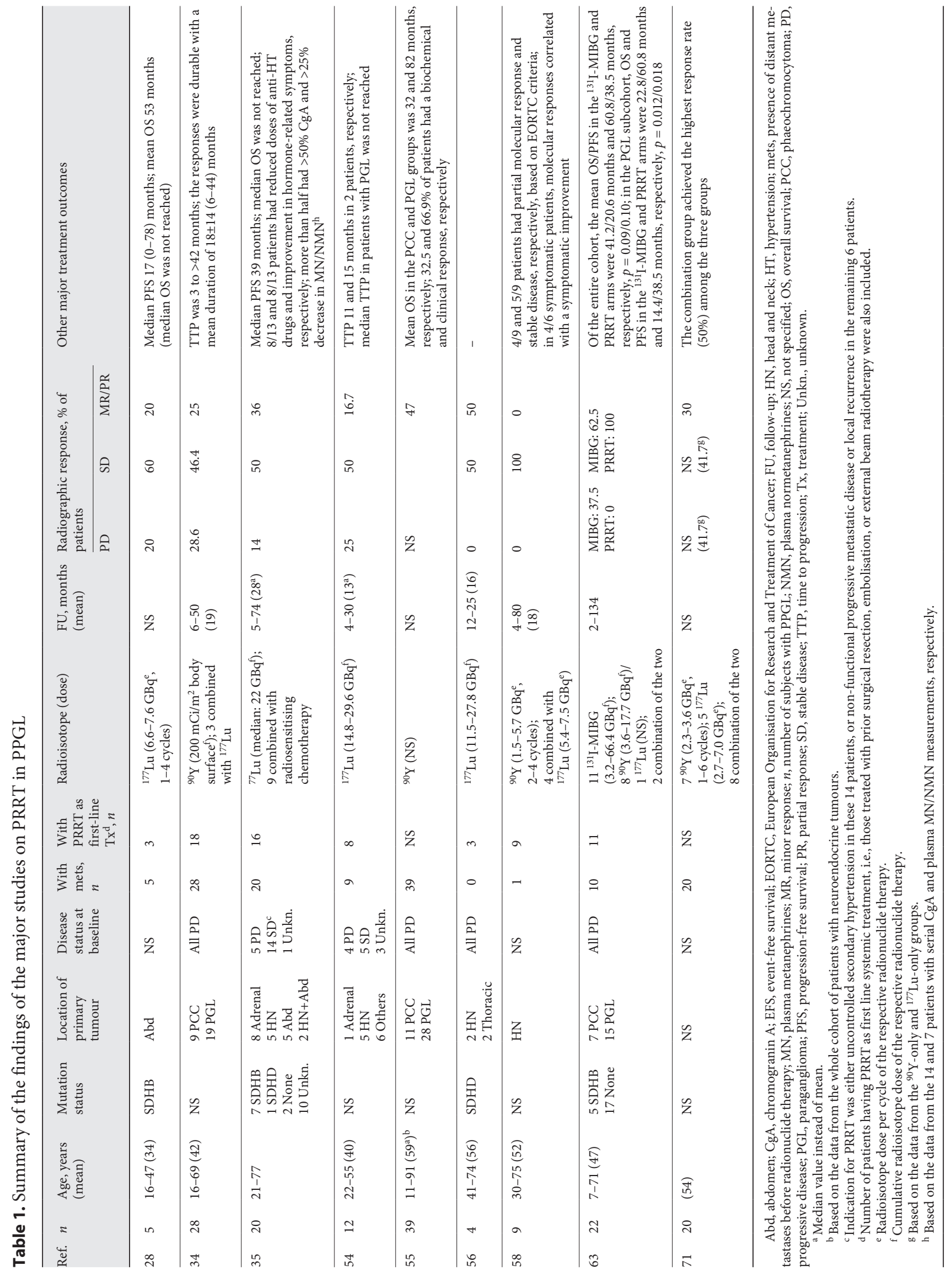


address this issue, which will need to consider cost, toxicity, and the accessibility of both types of radionuclide.

It is also unclear as to whether PRRT can be safely and effectively combined with radiosensitisers or chemotherapeutics $[35,72,73]$. The use of combinations of radiopharmaceuticals could also be explored for mixed SSTRand MIBG-avid lesions to allow possible synergistic effects and delivery of higher radiation doses to the respective target tissues with differential side effects [49]. Current advances in PRRT for NETs such as the use of radiolabelled SSTR antagonists, which are more readily bound to target cells [74], and high-energy a-particle emitters, may also be considered [75-77]. The findings of the major studies concerning the use of PRRT in PPGL are summarised in Table 1.

\section{Toxicity Concerns}

PRRT is usually well tolerated: a phase I study demonstrated the safety of PRRT co-administered with aminoacid infusion in 17 children and younger adults (1 PGL) [78]. Besides the known toxicity profile including fatigue, myelosuppression, and nephrotoxicity, a hormonal crisis precipitated by radionuclide therapy is a genuine concern in PPGL, as observed in a patient with a metastatic PCC who developed hypotension and myocardial ischaemia 1 day after ${ }^{177}$ Lu-DOTATATE therapy [79]. Makis et al. [80] also described 2 patients who developed an acute catecholamine crisis within hours and 3 days after treatment, respectively. We have also had experience of a catecholamine crisis in a patient with metastatic PCC during treatment with ${ }^{131}$ I-MIBG. Special precautions including adequate alpha-adrenoceptor blockade and close monitoring are mandatory when treating PPGL with PRRT, especially for syndromic patients [35], and this has also been our experience. It is possible that withholding glucocorticoid premedication [35] or lengthening the radioisotope infusion time and/or decreasing the initial treatment dose [80], may be helpful in preventing hypertensive crises. Certainly, close attention needs to be paid to fluctuations in blood-pressure control following each treatment. Pulmonary toxicity and intense pain related to reactive tumour swelling have also been reported [28].

\section{Limitations}

Some limitations of this review are recognised. As most of the available studies are small and retrospective in nature, the data provided are highly heterogeneous in terms of the clinical question of interest, patient population, study design and methodology, and treatment protocol. In addition, the definitions of tumour response (clinical, biochemical, radiological) and other parameters for survival analysis are inconsistent between the studies, rendering interpretation and comparison more difficult.

There were a wide range of indications for PRRT in these studies, such as presence of metastatic disease, prior disease progression, critical tumour location, uncontrolled hypertension, or a combination of the above. However, the treatment outcome may be variable in these patient groups. While many of the quoted studies principally included patients with metastatic lesions $[28,34,35$, 54, 55, 71], cases with non-metastatic PPGL were also reported in this review, comprising around $17 \%$ of the patients listed in Table 1. This may limit the overall interpretation of the potential efficacy of PRRT. Some studies predominantly included patients with progressive disease at baseline $[34,55,56,63]$, the target subgroup of patients who warrant further prospective research. However, a number of patients reported in this review had stable disease at study entry (Table 1), and such information was either unknown or lacking in the remaining cases. Of the 93 patients who had documented progressive disease prior to PRRT, a radiological tumour response was observed in $25-50 \%[34,54-56]$ with disease stabilisation in $71.4-$ $100 \%[34,35,54,56,63]$. However, we are unable to extrapolate these results to the rest of the study population.

It would be worthwhile to explore the role of PRRT as first-line treatment in progressive, metastatic PPGL. However, in the studies we reviewed, some subjects had undergone different treatment modalities prior to PRRT (Table 1), and we could not obtain specific outcome data in patients who had been treated with PRRT in the firstline setting.

We have been unable to provide sufficient evidence to demonstrate the possible difference in post-PRRT outcomes among various tumour subtypes (e.g., PCC vs. PGL) and different radionuclides ( ${ }^{131} \mathrm{I}-\mathrm{MIBG}$ vs. ${ }^{90} \mathrm{Y}-\mathrm{DO}$ TA-TOC/TATE vs. ${ }^{177} \mathrm{Lu}$-DOTA-TOC/TATE, alone or in combination) due to the very limited number of comparative studies. Further prospective clinical trials involving larger numbers of patients with metastatic PPGL should be able to explore these factors in more detail.

\section{Conclusions}

Although we believe there is an emerging role for PRRT in treating SSTR-positive metastatic PPGL, there are still many unanswered questions concerning the most appropriate therapeutic approach. Considering its favourable treatment outcome with minimal toxicity con- 
cern, and with the increasing availability of more effective and practical radionuclide agents, PRRT in its current or future guises looks to play a leading role in the therapy of these tumours; however, phase III randomised controlled trials are required to definitively establish the role of PRRT in the treatment of these very difficult cases. The role of newer targeted anti-oncogenic agents, alone and in combination, also needs to be taken into account in treating these complex tumours [81,82].

\section{Statement of Ethics}

The authors have no ethical conflicts to disclose.

\section{Disclosure Statement}

The authors have no conflicts of interest to declare.

\section{Funding Sources}

The authors have no relevant funding sources to declare.

\section{Author Contributions}

All authors see and manage patients with PPGL receiving PRRT. The first draft was written by the corresponding author while all authors contributed to writing and commenting on the final report.

\section{References}

1 Angelousi A, Kassi E, Zografos G, Kaltsas G. Metastatic pheochromocytoma and paraganglioma. Eur J Clin Invest. 2015 Sep;45(9):98697.

2 Fishbein L, Ben-Maimon S, Keefe S, Cengel K, Pryma DA, Loaiza-Bonilla A, et al. SDHB mutation carriers with malignant pheochromocytoma respond better to CVD. Endocr Relat Cancer. 2017 Aug;24(8):L51-5.

3 Lenders JW, Duh QY, Eisenhofer G, GimenezRoqueplo AP, Grebe SK, Murad MH, et al.; Endocrine Society. Pheochromocytoma and paraganglioma: an endocrine society clinical practice guideline. J Clin Endocrinol Metab. 2014 Jun;99(6):1915-42.

4 Grossman A, Pacak K, Sawka A, Lenders JW, Harlander D, Peaston RT, et al. Biochemical diagnosis and localization of phaeochromocytoma: can we reach a consensus? Ann N Y Acad Sci. 2006;1073(1):332-47.

5 Korevaar TI, Grossman AB. Pheochromocytomas and paragangliomas: assessment of malignant potential. Endocrine. 2011 Dec; 40(3):354-65.

6 Hamidi O, Young WF Jr, Iñiguez-Ariza NM, Kittah NE, Gruber L, Bancos C, et al. Malignant phaeochromocytoma and paraganglioma: 272 patients over 55 years. J Clin Endocrinol Metab. 2017 Sep;102(9):3296-305.

7 Lam AK. Update on adrenal tumours in 2017 World Health Organization (WHO) of endocrine tumours. Endocr Pathol. 2017 Sep; 28(3):213-27.

8 Pacak K, Eisenhofer G, Ahlman H, Bornstein SR, Gimenez-Roqueplo AP, Grossman AB, et al.; International Symposium on Pheochromocytoma. Pheochromocytoma: recommendations for clinical practice from the First International Symposium. October 2005. Nat Clin Pract Endocrinol Metab. 2007 Feb;3(2): 92-102.
9 Scholz T, Eisenhofer G, Pacak K, Dralle H, Lehnert $\mathrm{H}$. Clinical review: current treatment of malignant pheochromocytoma. J Clin Endocrinol Metab. 2007 Apr;92(4):1217-25.

10 Roman-Gonzalez A, Zhou S, Ayala-Ramirez M, Shen C, Waguespack SG, Habra MA, et al. Impact of surgical resection of the primary tumour on overall survival in patients with metastatic phaeochromocytoma or sympathetic paraganglioma. Ann Surg. 2018 Jul;268(1): 172-8.

11 Breen W, Bancos I, Young WF Jr, Bible KC, Laack NN, Foote RL, et al. External beam radiation therapy for advanced/unresectable malignant paraganglioma and pheochromocytoma. Adv Radiat Oncol. 2017 Nov;3(1): 25-9.

12 Niemeijer ND, Alblas G, van Hulsteijn LT, Dekkers OM, Corssmit EP. Chemotherapy with cyclophosphamide, vincristine and dacarbazine for malignant paraganglioma and pheochromocytoma: systematic review and meta-analysis. Clin Endocrinol (Oxf). 2014 Nov;81(5):642-51.

13 Huang H, Abraham J, Hung E, Averbuch S, Merino M, Steinberg SM, et al. Treatment of malignant pheochromocytoma/paraganglioma with cyclophosphamide, vincristine, and dacarbazine: recommendation from a 22-year follow-up of 18 patients. Cancer. 2008 Oct; 113(8):2020-8.

14 Jawed I, Velarde M, Därr R, Wolf KI, Adams $\mathrm{K}$, Venkatesan AM, et al. Continued tumour reduction of metastatic phaeochromocyto$\mathrm{ma}$ /paraganglioma harbouring succinate dehydrogenase subunit B mutations with cyclical chemotherapy. Cell Mol Neurobiol. 2018 Jul;38(5):1099-106.

15 Hadoux J, Favier J, Scoazec JY, Leboulleux S, Al Ghuzlan A, Caramella C, et al. SDHB mutations are associated with response to temo- zolomide in patients with metastatic pheochromocytoma or paraganglioma. Int J Cancer. 2014 Dec;135(11):2711-20.

16 Ayala-Ramirez M, Chougnet CN, Habra MA, Palmer JL, Leboulleux S, Cabanillas $\mathrm{ME}$, et al. Treatment with sunitinib for patients with progressive metastatic pheochromocytomas and sympathetic paragangliomas. J Clin Endocrinol Metab. 2012 Nov:97(11):4040-50.

17 Jimenez P, Tatsui C, Jessop A, Thosani S, Jimenez C. Treatment for malignant phaeochromocytomas and paragangliomas: 5 years of progress. Curr Oncol Rep. 2017 Oct; 19(12): 83.

18 Taïeb D, Timmers HJ, Hindié E, Guillet BA, Neumann HP, Walz MK, et al.; European Association of Nuclear Medicine. EANM 2012 guidelines for radionuclide imaging of phaeochromocytoma and paraganglioma. Eur J Nucl Med Mol Imaging. 2012 Dec;39(12): 1977-95.

19 Basu S, Abhyankar A, Jatale P. The current place and indications of 131I-metaiodobenzylguanidine therapy in the era of peptide receptor radionuclide therapy: determinants to consider for evolving the best practice and envisioning a personalized approach. Nucl Med Commun. 2015 Jan;36(1):1-7.

20 van Hulsteijn LT, Niemeijer ND, Dekkers OM, Corssmit EP. (131)I-MIBG therapy for malignant paraganglioma and phaeochromocytoma: systematic review and meta-analysis. Clin Endocrinol (Oxf). 2014 Apr;80(4):487501.

21 Loh KC, Fitzgerald PA, Matthay KK, Yeo PP, Price DC. The treatment of malignant pheochromocytoma with iodine-131 metaiodobenzylguanidine (131I-MIBG): a comprehensive review of 116 reported patients. J Endocrinol Invest. 1997 Dec;20(11):648-58. 
22 Yoshinaga $\mathrm{K}$, Oriuchi $\mathrm{N}$, Wakabayashi $\mathrm{H}$, Tomiyama Y, Jinguji M, Higuchi T, et al.; Drafting Committee for Guidelines on Internal Radiotherapy with ${ }^{131}$ I-MIBG; Japanese Society of Nuclear Medicine in Oncology and Imunology; Japanese Society of Nuclear Medicine. Effects and safety of ${ }^{131} \mathrm{I}$-metaiodobenzylguanidine (MIBG) radiotherapy in malignant neuroendocrine tumors: results from a multicenter observational registry. Endocr J. 2014;61(12):1171-80.

23 Fitzgerald PA, Goldsby RE, Huberty JP, Price DC, Hawkins RA, Veatch JJ, et al. Malignant pheochromocytomas and paragangliomas: a phase II study of therapy with high-dose 131Imetaiodobenzylguanidine (131I-MIBG). Ann N Y Acad Sci. 2006 Aug;1073(1):465-90.

24 Sze WC, Grossman AB, Goddard I, Amendra D, Shieh SC, Plowman PN, et al. Sequelae and survivorship in patients treated with (131)IMIBG therapy. Br J Cancer. 2013 Aug;109(3): 565-72.

25 Gonias S, Goldsby R, Matthay KK, Hawkins R, Price D, Huberty J, et al. Phase II study of high-dose [131I]metaiodobenzylguanidine therapy for patients with metastatic pheochromocytoma and paraganglioma. J Clin Oncol. 2009 Sep;27(25):4162-8.

26 Pryma DA, Chin BB, Noto RB, Dillon JS, Perkins S, Solnes L, et al. Efficacy and safety of high-specific-activity I-131 MIBG therapy in patients with advanced pheochromocytoma or paraganglioma. J Nucl Med. 2018. doi: 10.2967/jnumed.118.217463.

27 Van Der Horst-Schrivers AN, Jager PL, Boezen HM, Schouten JP, Kema IP, Links TP. Iodine-123 metaiodobenzylguanidine scintigraphy in localising phaeochromocytomas-experience and meta-analysis. Anticancer Res. 2006 Mar-Apr;26 2B:1599-604.

28 Pinato DJ, Black JR, Ramaswami R, Tan TM, Adjogatse D, Sharma R. Peptide receptor radionuclide therapy for metastatic paragangliomas. Med Oncol. 2016 May;33(5):47.

29 Alonso-Gordoa T, Capdevila J, Grande E. GEP-NETs update: biotherapy for neuroendocrine tumours. Eur J Endocrinol. 2015 Jan; 172(1):R31-46.

30 Hofman MS, Lau WF, Hicks RJ. Somatostatin receptor imaging with $68 \mathrm{Ga}$ DOTATATE PET/CT: clinical utility, normal patterns, pearls, and pitfalls in interpretation. Radiographics. 2015 Mar-Apr;35(2):500-16.

31 Reubi JC, Waser B, Khosla S, Kvols L, Goellner JR, Krenning E, et al. In vitro and in vivo detection of somatostatin receptors in pheochromocytomas and paragangliomas. J Clin Endocrinol Metab. 1992 May;74(5):1082-9.

32 Saveanu A, Muresan M, De Micco C, Taieb D, Germanetti AL, Sebag F, et al. Expression of somatostatin receptors, dopamine $\mathrm{D}_{2}$ receptors, noradrenaline transporters, and vesicular monoamine transporters in 52 pheochromocytomas and paragangliomas. Endocr Relat Cancer. 2011 Mar;18(2):287-300.
33 Mundschenk J, Unger N, Schulz S, Höllt V, Schulz S, Steinke R, et al. Somatostatin receptor subtypes in human pheochromocytoma: subcellular expression pattern and functional relevance for octreotide scintigraphy. J Clin Endocrinol Metab. 2003 Nov;88(11):5150-7.

34 Forrer F, Riedweg I, Maecke HR, MuellerBrand J. Radiolabeled DOTATOC in patients with advanced paraganglioma and pheochromocytoma. Q J Nucl Med Mol Imaging. 2008 Dec;52(4):334-40.

35 Kong G, Grozinsky-Glasberg S, Hofman MS, Callahan J, Meirovitz A, Maimon O, et al. Efficacy of peptide receptor radionuclide therapy for functional metastatic paraganglioma and phaeochromocytoma. J Clin Endocrinol Metab. 2017 Sep;102(9):3278-87.

36 Chang CA, Pattison DA, Tothill RW, Kong G, Akhurst TJ, Hicks RJ, et al. (68)Ga-DOTATATE and (18)F-FDG PET/CT in paraganglioma and pheochromocytoma: utility, patterns and heterogeneity. Cancer Imaging. 2016 Aug;16(1):22.

37 Björklund P, Pacak K, Crona J. Precision medicine in pheochromocytoma and paraganglioma: current and future concepts. J Intern Med. 2016 Dec;280(6):559-73.

38 Elston MS, Meyer-Rochow GY, Conaglen HM, Clarkson A, Clifton-Bligh RJ, Conaglen JV, et al. Increased SSTR2A and SSTR3 expression in succinate dehydrogenase-deficient pheochromocytomas and paragangliomas. Hum Pathol. 2015 Mar;46(3):390-6.

39 Janssen I, Blanchet EM, Adams K, Chen CC, Millo CM, Herscovitch P, et al. Superiority of [68Ga]-DOTATATEPET/CT to other functional imaging modalities in the localisation of SDHB-associated metastatic phaeochromocytoma and paraganglioma. Clin Cancer Res. 2015 Sep;21(17):3888-95.

40 Janssen I, Chen CC, Taieb D, Patronas NJ, Millo CM, Adams KT, et al. 68Ga-DOTATATE PET/CT in the localisation of head and neck paragangliomas compared with other functional imaging modalities and CT/MRI. J Nucl Med. 2016 Feb;57(2):186-91.

41 Taïeb D, Pacak K. New insights into the nuclear imaging phenotypes of cluster 1 phaeochromocytoma and paraganglioma. Trends Endocrinol Metab. 2017 Nov;28(11):807-17.

42 Han S, Suh CH, Woo S, Kim YJ, Lee JJ. Performance of $68 \mathrm{Ga}$-DOTA-conjugated somatostatin receptor targeting peptide PET in detection of phaeochromocytoma and paraganglioma: a systematic review and meta-analysis. J Nucl Med. 2018 Jul;60(3):369-76. https:// doi.org/10.2967/jnumed.118.211706.

43 Sharma P, Dhull VS, Arora S, Gupta P, Kumar R, Durgapal P, et al. Diagnostic accuracy of (68)Ga-DOTANOC PET/CT imaging in pheochromocytoma. Eur J Nucl Med Mol Imaging. 2014 Mar;41(3):494-504.
44 Tan TH, Hussein Z, Saad FF, Shuaib IL. Diagnostic performance of 68Ga-DOTATATE PET/CT, 18F-FDG PET/CT and 131I-MIBG scintigraphy in mapping metastatic phaeochromocytoma and paraganglioma. Nucl Med Mol Imaging. 2015 Jun;49(2):143-51.

45 Maurice JB, Troke R, Win Z, Ramachandran R, Al-Nahhas A, Naji M, et al. A comparison of the performance of ${ }^{68} \mathrm{Ga}$-DOTATATE $\mathrm{PET} / \mathrm{CT}$ and ${ }^{123} \mathrm{I}-\mathrm{MIBG}$ SPECT in the diagnosis and follow-up of phaeochromocytoma and paraganglioma. Eur J Nucl Med Mol Imaging. 2012 Aug;39(8):1266-70.

46 Lamarre-Cliche M, Gimenez-Roqueplo AP, Billaud E, Baudin E, Luton JP, Plouin PF. Effects of slow-release octreotide on urinary metanephrine excretion and plasma chromogranin A and catecholamine levels in patients with malignant or recurrent phaeochromocytoma. Clin Endocrinol (Oxf). 2002 Nov;57(5): 629-34.

47 Leijon H, PhL SR, Hagström J, Louhimo J, Mäenpää $\mathrm{H}$, Schalin-Jäntti C, et al. Variable somatostatin receptor subtype expression in 151 primary pheochromocytomas and paragangliomas. Hum Pathol. 2019 Apr;86:66-75.

48 Unger N, Serdiuk I, Sheu SY, Walz MK, Schulz S, Schmid KW, et al. Immunohistochemical determination of somatostatin receptor subtypes $1,2 \mathrm{~A}, 3,4$, and 5 in various adrenal tumors. Endocr Res. 2004 Nov;30(4): 931-4.

49 Chrisoulidou A, Kaltsas G, Ilias I, Grossman $\mathrm{AB}$. The diagnosis and management of malignant phaeochromocytoma and paraganglioma. Endocr Relat Cancer. 2007 Sep;14(3): 569-85.

50 Strosberg J, El-Haddad G, Wolin E, Hendifar A, Yao J, Chasen B, et al. Phase 3 trial of 177Lu-Dotatate for midgut neuroendocrine tumours. N Engl J Med. 2017 Jan;376(2):12535.

51 Strosberg J, Wolin E, Chasen B, Kulke M, Bushnell D, Caplin M, et al. Health-related quality of life in patients with progressive midgut neuroendocrine tumours treated with 177Lu-Dotatate in the phase III NETTER-1 trial. J Clin Oncol. 2018 Sep;36(25):2578-84.

52 Parus JL, Mikolajczak R. Beta-emitting radionuclides for peptide receptor radionuclide therapy. Curr Top Med Chem. 2012;12(23): 2686-93.

53 Frilling A, Weber F, Saner F, Bockisch A, Hofmann M, Mueller-Brand J, et al. Treatment with (90)Y- and (177)Lu-DOTATOC in patients with metastatic neuroendocrine tumors. Surgery. 2006 Dec;140(6):968-76.

54 van Essen M, Krenning EP, Kooij PP, Bakker WH, Feelders RA, de Herder WW, et al. Effects of therapy with [177Lu-DOTA0, Tyr3] octreotate in patients with paraganglioma, meningioma, small cell lung carcinoma, and melanoma. J Nucl Med. 2006 Oct;47(10): 1599-606. 
55 Imhof A, Brunner P, Marincek N, Briel M, Schindler C, Rasch H, et al. Response, survival, and long-term toxicity after therapy with the radiolabeled somatostatin analogue [90YDOTA]-TOC in metastasized neuroendocrine cancers. J Clin Oncol. 2011 Jun;29(17): 2416-23.

56 Zovato S, Kumanova A, Demattè S, Sansovini M, Bodei L, Di Sarra D, et al. Peptide receptor radionuclide therapy (PRRT) with $177 \mathrm{Lu}-$ DOTATATE in individuals with neck or mediastinal paraganglioma (PGL). Horm Metab Res. 2012 May;44(5):411-4.

57 Poeppel TD, Yuece A, Boy C, Metz KA, Kaminsky E, Neumann HP, et al. Novel SDHD gene mutation (H102R) in a patient with metastatic cervical paraganglioma effectively treated by peptide receptor radionuclide therapy. J Clin Oncol. 2011 Nov;29(33):e812-5.

58 Puranik AD, Kulkarni HR, Singh A, Baum RP. Peptide receptor radionuclide therapy with (90)Y/ (177)Lu-labelled peptides for inoperable head and neck paragangliomas (glomus tumours). Eur J Nucl Med Mol Imaging. 2015 Jul;42(8):1223-30.

59 Gupta SK, Singla S, Karunanithi S, Damle N, Bal C. Peptide receptor radionuclide therapy with (177)Lu DOTATATE in a case of recurrent carotid body paraganglioma with spinal metastases. Clin Nucl Med. 2014 May;39(5): $440-1$.

60 Cecchin D, Schiavi F, Fanti S, Favero M, Manara R, Fassina A, et al. Peptide receptor radionuclide therapy in a case of multiple spinal canal and cranial paragangliomas. J Clin Oncol. 2011 Mar;29(7):e171-4.

61 Estêvão R, Duarte H, Lopes F, Fernandes J, Monteiro E. Peptide receptor radionuclide therapy in head and neck paragangliomas Report of 14 cases. Rev Laryngol Otol Rhinol (Bord). 2015;136(4):155-8.

62 Casey RT, McLean MA, Madhu B, Challis BG, ten Hoopen R, Roberts T, et al. Translating in vivo metabolomic analysis of succinate dehydrogenase-deficient tumours into clinical utility. JCO Precis Oncol. 2018;2:1-12.

63 Nastos K, Cheung VT, Toumpanakis C, Navalkissoor S, Quigley AM, Caplin M, et al. Peptide Receptor Radionuclide Treatment and (131)I-MIBG in the management of patients with metastatic/progressive phaeochromocytomas and paragangliomas. J Surg Oncol. 2017 Mar;115(4):425-34.
64 Cives M, Strosberg J. Radionuclide therapy for neuroendocrine tumours. Curr Oncol Rep. 2017 Feb;19(2):9.

65 Bodei L, Mueller-Brand J, Baum RP, Pavel ME, Hörsch D, O’Dorisio MS, et al. The joint IAEA, EANM, and SNMMI practical guidance on peptide receptor radionuclide therapy (PRRNT) in neuroendocrine tumours. Eur J Nucl Med Mol Imaging. 2013 May;40(5): 800-16.

66 Romer A, Seiler D, Marincek N, Brunner P, Koller MT, Ng QK, et al. Somatostatin-based radiopeptide therapy with [177Lu-DOTA]TOC versus [90Y-DOTA]-TOC in neuroendocrine tumours. Eur J Nucl Med Mol Imaging. 2014 Feb;41(2):214-22.

67 Gulenchyn KY, Yao X, Asa SL, Singh S, Law C. Radionuclide therapy in neuroendocrine tumours: a systematic review. Clin Oncol (R Coll Radiol). 2012 May;24(4):294-308.

68 Nicolini S, Sansovini M, Bongiovanni A, Paganelli G. Peptide receptor radionuclide therapy in the management of gastrointestinal neuroendocrine tumours: efficacy profile, safety, and quality of life. Onco Targets Ther. 2017; 10:551-7.

69 Seregni E, Maccauro M, Chiesa C, Mariani L, Pascali C, Mazzaferro V, et al. Treatment with tandem [90Y]DOTA-TATE and [177Lu]DOTA-TATE of neuroendocrine tumours refractory to conventional therapy. Eur J Nucl Med Mol Imaging. 2014 Feb;41(2):223-30.

70 Villard L, Romer A, Marincek N, Brunner P, Koller MT, Schindler C, et al. Cohort study of somatostatin-based radiopeptide therapy with [(90)Y-DOTA]-TOC versus [(90)YDOTA]-TOC plus [(177)Lu-DOTA]-TOC in neuroendocrine cancers. J Clin Oncol. 2012 Apr;30(10):1100-6.

71 Prasad V, Zachert C, Schuchardt C, Wortmann R, Baum R. Peptide receptor radionuclide therapy (PRRT) for progressive, somatostatin receptor positive phaeochromocytoma/paraganglioma. J Nucl Med. 2008;49 Suppl 1:101P.

72 Ullrich M, Bergmann R, Peitzsch M, Zenker EF, Cartellieri M, Bachmann M, et al. Multimodal somatostatin receptor theranostics using $[64 \mathrm{Cu}] \mathrm{Cu}-/[177 \mathrm{Lu}] \mathrm{Lu}-$ DOTA-(Tyr3)octreotate and $\mathrm{AN}-238$ in a mouse phaeochromocytoma model. Theranostics. 2016 Mar; 6(5):650-65.
73 Ashwathanarayana AG, Biswal CK, Sood A, Parihar AS, Kapoor R, Mittal BR. Imagingguided use of combined 177Lu-DOTATATE and capecitabine therapy in metastatic mediastinal paraganglioma. J Nucl Med Technol. 2017 Dec;45(4):314-6.

74 Fani M, Nicolas GP, Wild D. Somatostatin receptor antagonists for imaging and therapy. J Nucl Med. 2017 Sep;58 Suppl 2:61S-6S.

75 Makvandi M, Dupis E, Engle JW, Nortier FM, Fassbender ME, Simon S, et al. Alpha-emitters and targeted alpha therapy in oncology: from basic science to clinical investigations. Target Oncol. 2018 Apr;13(2):189-203.

76 Kratochwil C, Giesel FL, Bruchertseifer F, Mier W, Apostolidis C, Boll R, et al. ${ }^{213} \mathrm{Bi}-$ DOTATOC receptor-targeted alpha-radionuclide therapy induces remission in neuroendocrine tumours refractory to beta radiation: a first-in-human experience. Eur J Nucl Med Mol Imaging. 2014 Nov;41(11):2106-19.

77 Grossman A, Navalkissoor S. Targeted Alpha Particle Therapy for Neuroendocrine Tumours: The Next Generation of Peptide Receptor Radionuclide Therapy. Neuroendocrinology. 2018 Oct. doi: https://doi. org/10.1159/000494760.

78 Menda Y, O’Dorisio MS, Kao S, Khanna G, Michael S, Connolly M, et al. Phase I trial of 90Y-DOTATOC therapy in children and young adults with refractory solid tumors that express somatostatin receptors. J Nucl Med. 2010 Oct;51(10):1524-31.

79 de Keizer B, van Aken MO, Feelders RA, de Herder WW, Kam BL, van Essen M, et al. Hormonal crises following receptor radionuclide therapy with the radiolabeled somatostatin analogue [177Lu-DOTA0,Tyr3]octreotate. Eur J Nucl Med Mol Imaging. 2008 Apr;35(4):749-55.

80 Makis W, McCann K, McEwan AJ. The challenges of treating paraganglioma patients with 177Lu-DOTATATE PRRT: catecholamine crises, tumour lysis syndrome and the need for modification of treatment protocols. Nucl Med Mol Imaging. 2015 Sep;49(3):22330.

81 Jochmanova I, Pacak K. Genomic landscape of phaeochromocytoma and paraganglioma. Trends Cancer. 2018 Jan;4(1):6-9.

82 Nolting S, Grossman A, Pacak K. Metastatic phaeochromocytoma: spinning towards more promising treatment options. Exp Clin Endocrinol Diabetes. 2019;127:117-128. https://doi.org/10.1055/a-0715-1888. 